\title{
A new method for resolution of discontinuities with changes in sediment transport form in a numerical simulation
}

\author{
Takuro SUZUKI ${ }^{1}$, Taro UCHIDA ${ }^{2}$ and Atsushi OKAMOTO ${ }^{2}$ \\ ${ }^{1}$ Forestry and Forest Products Research Institute (1 Matsunosato, Tsukuba, Ibaraki 3058687, Japan) \\ E-mail: takurosuzuki@ffpri.affrc.go.jp \\ 2 National Institute for Land and Infrastructure Management (1 Asahi, Tsukuba, Ibaraki 3058572, Japan)
}

\begin{abstract}
To deal with transitional flow from debris flow through sheet flow to bedload flow, a riverbed shear stress formula was developed by applying the existing theory of debris flow to the sediment layer and Manning's theory to the water layer. We also propose a new method for determining the transitional condition of the sediment transport region to a continuous transition of equilibrium concentration. Numerical simulations were performed using an existing model and our new model. Results for conditions where the riverbed gradient changed gradually showed that the existing model indicated irregular deposition deformation. Moreover, simulation results of flow depth and sediment concentration oscillated around transition boundaries. When discontinuities of riverbed shear stress and equilibrium sediment concentration coincided, numerical oscillations became larger. Our model yielded smooth and stable simulation results without these problems.
\end{abstract}

Key words: Debris flow, bedload, numerical simulation, continuity around transition boundary

\section{INTRODUCTION}

It is important to deal with transitional flow from debris flow through sheet flow to bedload flow to estimate the effect of debris flows on downstream area. Egashira et al. [1990] developed a two-layer model of a sediment layer and a water layer for sheet flow (the Egashira model). Constitutive equations for debris flow were used for the sediment layer and a logarithmic velocity distribution based on the mixing length theory of Prandtl was introduced for the water layer. Based on this model, Takahama et al. [2000] developed a simulation method for two-layer flow, solving the continuity equation and the momentum equation of each layer. Miyamoto and Itoh [2002] developed a one -layer simulation method with the riverbed sheer stress equation, derived by integrating the velocity distribution from the sediment layer into the water layer.

Egashira et al. [1997] showed that the two-layer model could deal rationally with transitional flow from debris flow through sheet flow to bedload flow. Thus, a simulation method based on Egashira et al. [1990] has become an established method for determining transitional flow from debris flow to bedload flow.

The Manning equation is used widely for resistance law of bed deformation calculations of bedload and the
Manning coefficient is a parameter of riverbed roughness [e. g., Japan Society of Erosion Control Engineering, 2000]. The Egashira model does not agree with the Manning equation. In the water layer of the Egashira model, the virtual origin of velocity distribution is evaluated using a void scale of the interparticles of the sediment layer. This means that the Egashira model includes no condition regarding riverbed roughness.

In contrast, Takahashi et al. [1991, 1996] (the Takahashi model) classified flow type into debris flow, sheet flow, and bedload, depending on sediment concentration. The riverbed sheer stress formula and the equilibrium sediment concentration formula were defined for each flow type. With this model, many numerical simulations have been conducted [e. g. Nakagawa et al., 2001] and software packages were developed [e. g. Nakatani, 2010]. However, transitional points of flow types may be discontinuous in the Takahashi model because their dependence on flow depth and sediment diameter differ among the formulae for each flow type. This discontinuity may cause numerical oscillations or numerical results with abrupt changes in flow depth or deposition depth. This can lead to overestimations or underestimations of the effects of debris flows.

The aim of this study was to develop a stable 
simulation method for transitional flow from debris flow through sheet flow to bedload flow that includes a bedload model introducing the Manning equation and a bedload discharge formula, as in the widely used bed deformation calculation of bedload. For this purpose, we adopted the Takahashi model and analyzed discontinuity conditions with it. We then developed a stable model by modifying discontinuities in the sheer stress formula for sheet flow and the equilibrium sediment concentration formulae. The model developed was examined in terms of reproducibility of existing experimental results and stability in numerical simulations.

\section{EXISTING MODEL AND OBJECTIVE OF THIS STUDY}

\subsection{Numerical Simulation Method of Takahashi}

The numerical simulation method of Takahashi et al. [1991, 1996] is described as follows. Based on the shallow water equation, the governing equations consist of the momentum equation, the continuity equation for the total volume of debris flow, and the continuity equation for the particles. The vertical distribution of sediment concentration is treated as uniform, as in common numerical simulation methods.

The continuity equation for the total volume of the debris flow is written as

$$
\frac{\partial h}{\partial t}+\frac{\partial q}{\partial x}=E
$$

The momentum equation is written as

$$
\frac{\partial q}{\partial t}+\beta \frac{\partial(U q)}{\partial x}=-g h \frac{\partial H}{\partial x}-\frac{\tau_{0}}{\rho_{m}}
$$

The continuity equation for the particles is written as

$$
\frac{\partial(c h)}{\partial t}+\frac{\partial(c q)}{\partial x}=E c_{*}
$$

In Eqs. (1)-(3), $h$ is the flow depth, $q$ is the discharge per unit width, $U$ is the flow velocity, $g$ is the acceleration due to gravity, $\rho_{m}$ is the density of the debris flow, $H=h+z_{b}, z_{b}$ is the bed elevation, $E$ is the entrainment rate or the deposition rate, $\tau_{0}$ is the riverbed shear stress, $c$ is the average sediment concentration, and $c^{*}$ is the sediment concentration of the deposition layer. $E$ and $\tau_{0}$ are defined for each flow type. The riverbed shear stress formulae are written as

$c \geq 0.4 c_{*}$ (debris flow $)$

$$
\tau_{0}=\frac{25}{4} \rho K(c)\left(\frac{h}{d}\right)^{-2} U^{2}
$$

$$
K(c)=a_{i} \sin \alpha\left\{\left(\frac{c_{*}}{c}\right)^{\frac{1}{3}}-1\right\}^{-2} \frac{\sigma}{\rho}
$$

$0.01<c<0.4 c_{*}$ (sheet flow)

$$
\tau_{0}=\frac{\rho_{m}}{0.49}\left(\frac{h}{d}\right)^{-2} U^{2}
$$

$c \leq 0.01$ (bedload)

$$
\tau_{0}=\frac{\rho_{m} g n^{2} U^{2}}{h^{\frac{1}{3}}}
$$

In Eqs. (4) $-(7), d$ is the grain diameter, $\rho$ is the density of the pore fluid, $n$ is the Manning coefficient, $a_{i}$ is an empirical constant, $\alpha$ is the collision angle, and $a_{i} \cdot \sin \alpha$ is 0.02 .

The entrainment and deposition rate formulae can be written as

$c_{\infty} \geq c$ (entrainment)

$$
E=\delta_{e} \frac{c_{\infty}-c}{c_{*}-c_{\infty}} \frac{q}{d}
$$

$c_{\infty}<c$ (deposition)

$$
E=\delta_{d} \frac{c_{\infty}-c}{c_{*}} \frac{q}{h}
$$

In Eqs. (8) and (9), $\delta_{e}$ is the coefficient of entrainment and $\delta_{d}$ is the coefficient of deposition. $c_{\infty}$ is the equilibrium sediment concentration and is defined for each flow type.

The equilibrium sediment concentration formulae are written as

$\tan \theta_{w} \geq \tan \psi_{s}$

$$
c_{\infty}=0.9 c_{*}
$$

$\tan \psi_{s}>\tan \theta_{w}>0.138$ (debris flow)

$$
c_{\infty}=\frac{\rho \tan \theta_{w}}{(\sigma-\rho)\left(\tan \phi_{s}-\tan \theta_{w}\right)}
$$

$0.138 \geq \tan \theta_{w}>0.03$ (sheet flow)

$$
c_{\infty}=6.7\left\{\frac{\rho \tan \theta_{w}}{(\sigma-\rho)\left(\tan \phi_{s}-\tan \theta_{w}\right)}\right\}^{2}
$$

$\tan \theta_{w} \leq 0.03$ (bedload)

$$
\begin{gathered}
c_{\infty}=\frac{\left(1+5 \tan \theta_{w}\right) \tan \theta_{w}}{\frac{\sigma}{\rho}-1} \\
\left(1-\alpha_{c}{ }^{2} \frac{\tau_{* c}}{\tau_{*}}\right)\left(1-\alpha_{c} \sqrt{\frac{\tau_{* c}}{\tau_{*}}}\right) \\
\tau_{* c}=0.04 \times 10^{1.72 \tan \theta_{w}} \\
\alpha_{c}{ }^{2}=2\left(0.425-\frac{\sigma \tan \theta_{w}}{\sigma-\rho}\right)
\end{gathered}
$$




$$
\begin{array}{r}
/\left(1-\frac{\sigma \tan \theta_{w}}{\sigma-\rho}\right) \\
\tau_{*}=\frac{\rho}{\sigma-\rho} \frac{h \tan \theta_{w}}{d}
\end{array}
$$

When $\tau^{*} \leq \tau^{*}, c_{\infty}$ is zero. Here, $\theta_{w}$ is the slope of water surface in the flow direction, $\phi_{s}$ is the friction angle, $\tau^{*}$ is the dimensionless tractive force, and $\tau^{*}$ is the dimensionless critical tractive force.

\subsection{Discontinuities in the Takahashi model}

To discuss discontinuities among the riverbed sheer stress formulae, we focused on the coefficient of $U^{2}$. Equations (4) (debris flow formula) and (6) (sheet flow formula) have the same forms of $h$ and $d$; however, their dependences on $\sigma, \rho$, and $c$ vary. Thus, Eqs. (4) and (6) are continuous only with $\sigma=2.6$ and $\rho=1.0$, and they are almost always discontinuous with other values. The values of $\sigma$ and $\rho$, with which Eqs. (4) and (6) are continuous, depend on the border value of sediment concentration between debris flow and sheet flow. In the Takahashi model, this border value is 0.4 $c^{*}$, whereas it is $0.5 c^{*}$ in the Egashira model. Eq. (7) (bedload formula) and Eq. (6) are entirely different and basically discontinuous. Takahashi et al. [1988] derived Eq. (6) as an empirical formula from experimental results in previous studies. Takahashi [2004] compared results with this formula and the experimental results of Takahashi [1982] and Itoh [2000]. All experimental results were distributed around the line of Eq. (6). However, when examined more closely, the experimental results became independent of $h / d$ as the incline became lower. This indicated that the resistance law became closer to the Manning equation (turbulent flow) when the incline was lower. This was thought to be due to the water layer being more dominant to the resistance law when the sediment concentration is lower. Thus, Eq. (6) would seem to be inappropriate for low-incline conditions.

The equilibrium sediment concentration formulae for debris flow (Eq. (11)) and sheet flow (Eq. (12)) are both functions of $\sigma, \rho, \theta_{w}$, and $\phi_{s}$, but they have different forms. However, the border value of $\tan \theta_{w}$ between debris flow and sheet flow is a fixed value (= 0.138). Eqs. (11) and (12) are continuous only with $\sigma$ $=2.6, \rho=1.0$, and $\phi_{s}=35$, and they are almost always discontinuous with other values. Equation (13) (bedload formula) and Eq. (12) are entirely different and basically discontinuous. Takahashi [2004] compared the results of Eq. (12) with experimental results and indicated that they were generally in good agreement. However, the experimental results were slightly larger than those with Eq. (12).

\subsection{Objective}

In this study, we assumed that the formulae for debris flows and bedload were correct because they have been validated in many studies. Thus, we modified the riverbed sheer stress formula for sheet flow so that it transitions continuously from a sediment layer-dominating state to a water layerdominating state, depending on sediment concentration. For the equilibrium sediment concentration formulae, we developed a method in which the border values were variable for continuous transactions.

The modified models were examined by comparison with the experimental results of Takahashi [1982] and Itoh [2000] around the region of sheet flows. Moreover, numerical simulations were conducted to confirm the applicability and numerical stability of our model.

\section{DEVELOPMENT OF THE MODIFIED METHOD}

\subsection{Modified Sheer Stress Formula}

In this section, we describe the modified riverbed sheer stress formula. For sheet flow, we adopted the two-layer model and the resistance law of turbulent flows was applied to the water layer. Takahashi [1982] and Egashira et al. [1990] applied a logarithmic velocity distribution for the water layer, whereas we adopt the Manning equation for continuity with the bedload region. Figure 1 shows a schematic of the two-layer model. The height from the bed to the surface of the sediment layer is $h_{s}$ and the sediment concentration of the sediment layer, $c_{s}$, is constant. $c_{s}$ is expressed using $c^{*}$ and the coefficient $\gamma$ as

$$
c_{s}=\gamma c_{*}
$$

$h_{s}$ is expressed as

$$
h_{s}=h \frac{c}{c_{s}}
$$

We assume that sediment layer has the velocity

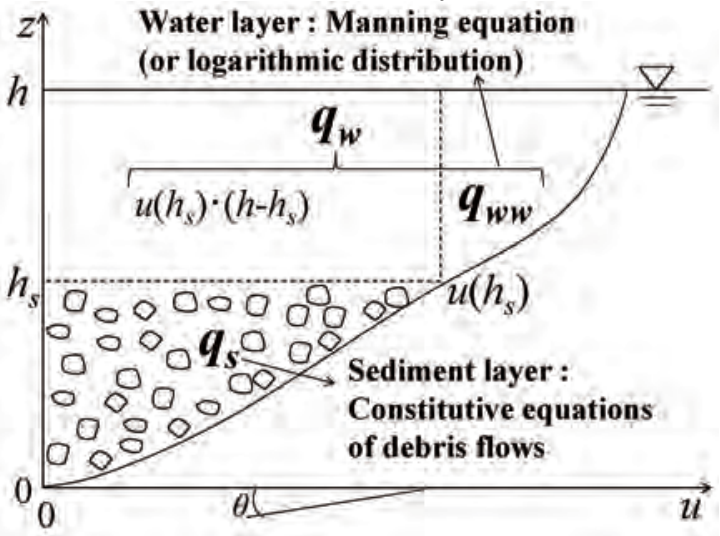

Fig. 1 Schematic of two-layer flow 
distribution of the debris flow and the water layer has that of the water. Integrating the flow velocity at $z, u$ (z), from bed to surface, $q$ is obtained.

$$
q=\int_{0}^{h} u(z) \cdot d z
$$

Here, $q$ can be divided into $q_{s}$ and $q_{w}$ (Fig. 1).

$$
q=q_{s}+q_{w}
$$

$q_{s}$ and $q_{w}$ correspond to the discharge of the sediment layer and the water layer, respectively. The velocity distribution of the sediment layer is obtained as follows. Eq. (21) is the balance equation of the sheer force and the sheer stress at $z$.

$$
\tau(z)=\int_{z}^{h} \rho_{m} g \sin \theta \cdot d z
$$

Substituting the sheer stress formula of Takahashi et al. [1991], Eq. (22), into Eq. (21), Eq. (23) is obtained.

$$
\begin{aligned}
& \tau(z)=\rho K(c) d^{2}\left(\frac{\partial u}{\partial z}\right)^{2} \\
& {\left[\rho(h-z)+\sigma c_{s}\left(h_{s}-z\right)\right] g \sin \theta} \\
& \quad=\rho K(c) d^{2}\left(\frac{\partial u}{\partial z}\right)^{2}
\end{aligned}
$$

$u(z)$ is obtained by deforming and integrating Eq. (23), and $q_{s}$ is calculated by substituting $u(z)$ into Eq. (24).

$$
q_{s}=\int_{0}^{h s} u(z) \cdot d z
$$

$q_{w}$ is calculated with Eq. (25).

$$
q_{w}=u\left(h_{s}\right) \cdot\left(h-h_{s}\right)+q_{w w}
$$

Here, $q_{w w}$ is the component corresponding to the change in the velocity distribution of the water flow and $q_{w}$ is the base component corresponding to the boundary velocity (Fig. 1). In our model, $q_{w w}$ is evaluated using the Manning equation as

$$
q_{w w}=\frac{\sqrt{\sin \theta}}{n}\left(h-h_{s}\right)^{\frac{5}{3}}
$$

The formula indicating the relationship between $U$ and the velocity gradient at the riverbed is obtained by substituting $z=0$ into Eqs. (21) and (22) and solving them with Eqs. (23)-(26). Substituting this formula and $z=0$ into Eq. (22), the formulae for $\tau_{0}$ are obtained as follows.

$$
\begin{gathered}
\tau_{0}=\rho K\left(c_{s}\right) r\left(\phi_{s i}+\phi_{w}\right)^{-2}\left(\frac{h}{d}\right)^{-2} U^{2} \\
\phi_{s i}=\frac{2}{3} r_{s}{ }^{-2}\left[\left(r_{s}-\frac{2}{5} r\right) r^{\frac{3}{2}}-\left(r_{s}-\frac{2}{5}\right) r_{w} \frac{5}{2}\right]
\end{gathered}
$$

$$
\begin{gathered}
\psi_{w}=\sqrt{\frac{K\left(c_{s}\right)}{g} \frac{h^{\frac{1}{6}}}{n}} r_{w}^{\frac{5}{3}}\left(\frac{h}{d}\right)^{-1} \\
r=1+c \frac{\sigma}{\rho} \\
r_{s}=1+c_{s} \frac{\sigma}{\rho} \\
r_{w}=1-\frac{c}{c_{s}}
\end{gathered}
$$

These formulae correspond to the Manning equation when $c$ is zero and the debris flow formula when $c$ is $c_{s}$. That means these formulae can express transitional flow from debris flow through sheet flow to bedload flow by adding the condition of $c_{s}=c$ for $c>c_{s}$. Additionally, when we apply a logarithmic velocity distribution for the water layer, Eq. (33) can be substituted into $\phi_{w}$.

$$
\begin{gathered}
\phi_{w}=\frac{1}{\kappa} \sqrt{K\left(c_{s}\right)} \cdot r_{w}^{\frac{1}{2}} \\
\left(\frac{\frac{l_{0}}{n}+\kappa r_{w}}{\kappa} \ln \frac{\frac{l_{0}}{n}+\kappa r_{w}}{\kappa}-r_{w}\right)\left(\frac{h}{d}\right)^{-1} \\
l_{0}=\sqrt{k_{f}}\left(\frac{1-c_{s}}{c_{s}}\right)^{\frac{1}{3}} d
\end{gathered}
$$

Here, $\kappa$ is the Karman constant. Equation (34) expresses the mixing length [Egashira et al., 1990] and $k_{f}$ is the empirical constant.

\subsection{Modified Equilibrium Sediment Concentration Model}

In this section, we describe the modified method for the equilibrium sediment concentration formulae. In our model, the boundary conditions are not constant but instead vary for continuous transactions. $\theta_{w 1}$ and $\theta_{w 2}$ are defined as the boundary surface gradient of the debris flow and sheet flow and that of the sheet flow and bedload, respectively. $\theta_{w 1}$ is given when Eq. (11) is equal to Eq. (12), as

$$
\tan \theta_{w 1}=\frac{(\sigma-\rho) \tan \phi_{s}}{\sigma+5.7 \rho}
$$

$\theta_{w 2}$ is given when Eq. (12) is equal to Eq. (13); however, it cannot be solved mathematically. Thus, it was solved numerically in simulations. There are some cases of $\theta_{w 1}<\theta_{w 2}$ depending on parameters. In these cases, flow state transits from debris flow to bedload directly at $\theta_{w 3}$, corresponding to $\theta_{w}$ when Eq. (11) is equal to Eq. (13). 


\subsection{Comparison with Takahashi Model in Continuities}

We investigated continuity in our model. To examine the sheer stress formulae, we focused on the relationship between the sediment concentration and the velocity coefficient, $U / u^{*}$, in an equilibrium state. We compared the calculated values of $U / u^{*}$ using Takahashi's model and our model. In our model, the resistance law of the water layer was basically the Manning equation (modified sheer stress model). In this section, we also compared the method when a logarithmic velocity distribution was applied to our model (Eq.33 ; logarithmic model). Figure 2 shows the results of four cases with different values of $h / d, n$, and $c_{s}$. In all cases, $\sigma=2.6, \rho=1.0$, and $h=1.0$ are fixed conditions. Focusing on the boundary of sheet flow and bedload $(c=0.01)$, the sheet flow formula changes with $h / d$ (Fig. 2 (a), (b)) and the bedload formula changes with $n$ (Fig. 2 (c)). Thus, they are basically discontinuous. At the boundary of debris flow and sheet flow $\left(c=c_{s}\right)$, the two formulae are almost continuous with $c_{s}=0.24$, but they are discontinuous with $c_{s}=0.30$. As above, Takahashi's model includes some discontinuities because the formulae for each flow state have different dependences on the parameters. In contrast, these discontinuities are resolved in the modified sheer stress

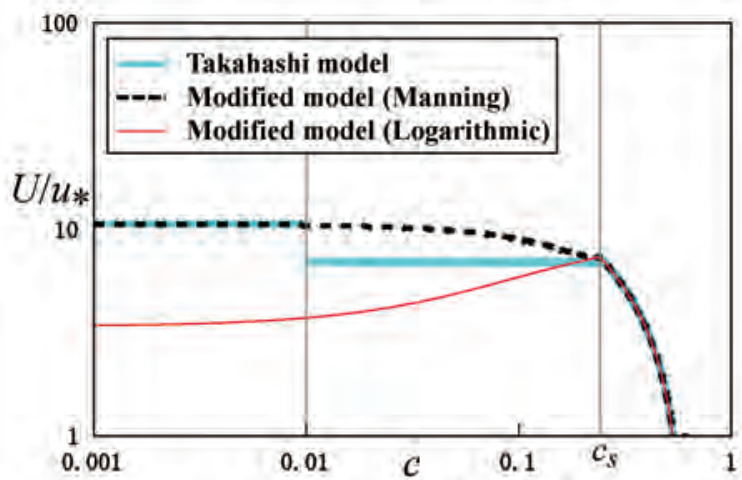

(a) $h / d=10, n=0.03, c_{s}=0.24$

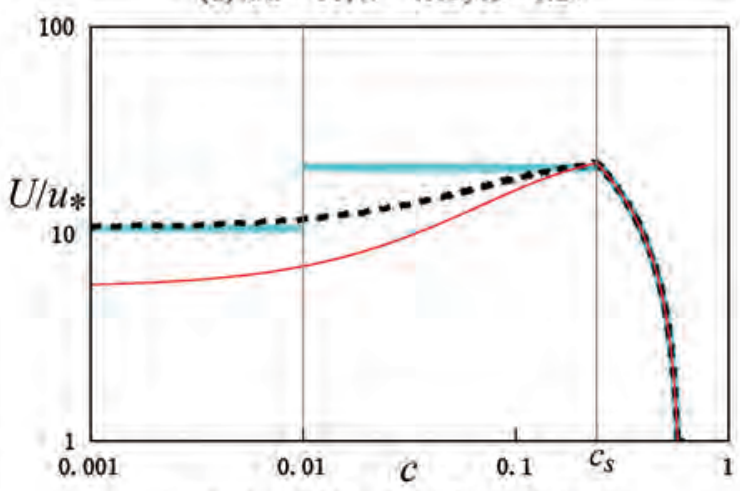

(b) $h / d=30, n=0.03, c_{x}=0.24$ model. The logarithmic model also resolves the discontinuities but does not agree with the Manning equation. To examine the equilibrium sediment concentration formulae, we focused on the relationship between $\tan \theta_{w}$ and $c_{\infty}$. Figure 3 shows the results of four cases with different values of $h / d$ and $\phi_{s}$. In all cases, $\sigma=2.6, \rho=1.0$ and $h=1.0$ are fixed conditions. Figures 3 (a) and 3 (b) are the results of conditions when the sheet flow formula and the debris flow formula are continuous at $\tan \theta_{w}=0.138$. The results of Takahashi's model are discontinuous at the boundary of sheet flow and bedload $\left(\tan \theta_{w}=0.03\right)$. In contrast, the results of our method (modified concentration model) show continuities and $\theta_{w 1}$ varies with $h / d$. Figure 3 (c) is the result of $\phi_{s}=30$. In this case, $\theta_{w 1}$ is lower than that of $\phi_{s}=35$ (Fig. 3 (a)) because the result of the sheet flow formula becomes larger when $\phi_{s}$ is small. Moreover, the results of Takahashi's model show the discontinuities at $\tan \theta_{w}=0.138$ in contrast to $\phi_{s}=35$.

This is because the result of the sheet flow formula varies more than that of the debris flow formula with a change in $\phi_{s}$. The results of the modified concentration model show continuities and $\tan \theta_{w 2}$ is lower than 0.138 . Figure 3 (d) is the result of $\phi_{s}=40$, and it shows the opposite result to the case of $\phi_{s}=30$. In this case, the result of the modified concentration model

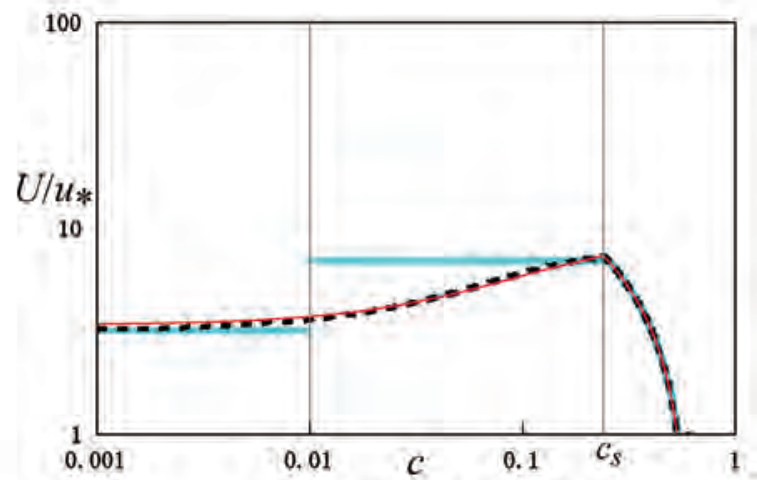

(c) $h / d=10, n=0.1, c_{s}=0.24$

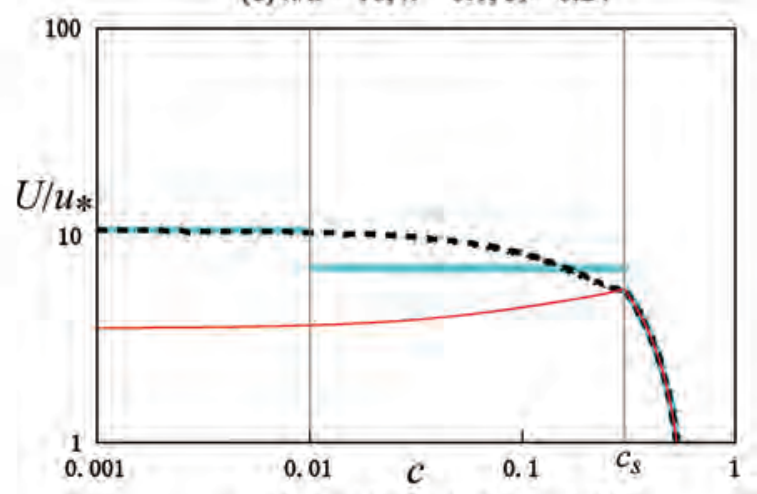

(d) $h / d=10, n=0.03, c_{s}=0.30$

Fig. 2 Continuity in riverbed shear stress 


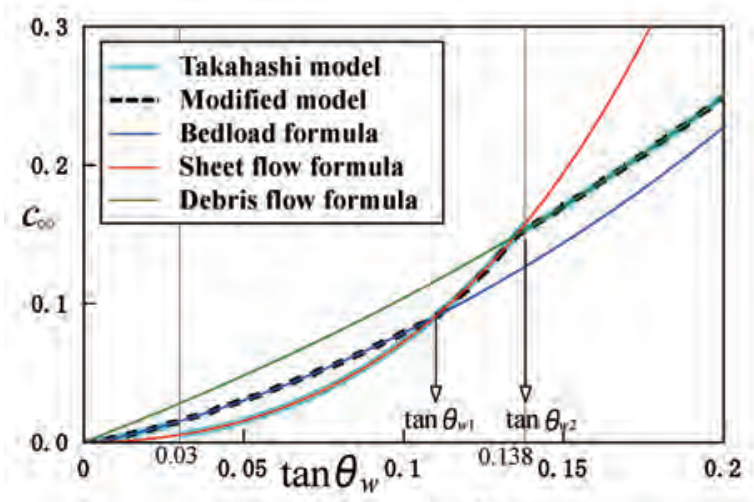

(a) $h / d=30, \varphi_{s}=35$

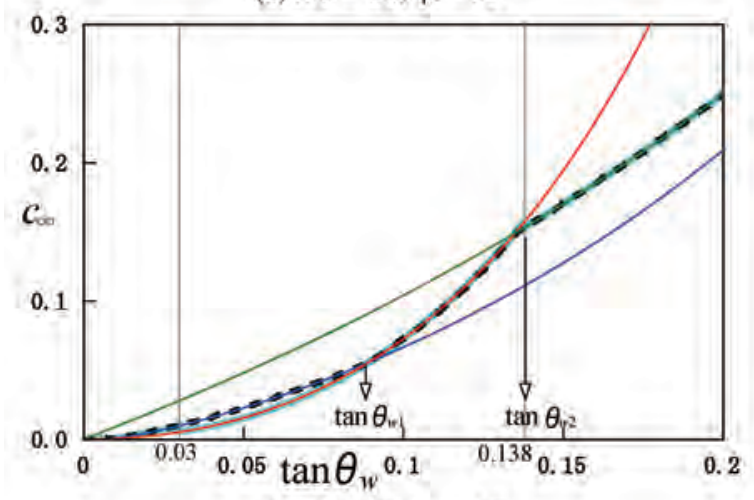

(b) $h / d=10, \varphi_{s}=35$

Fig. 3 Continuity in equilibrium sediment concentration

transits continuously and directly from debris flow to bedload because $\theta_{w 1}$ is larger than $\theta_{w 2}$.

\section{COMPARISON WITH EXPERIMENTS}

\subsection{Experimental Methods of Past Studies}

The modified sheer stress and concentration models were examined by comparison with experimental results of past studies around the region of sheet flow. We used the experimental results of Takahashi [1982] and Itoh [2000]. A variable slope channel was used for the experiment in Takahashi [1982]. Its length was 6 $\mathrm{m}$ and the width was $10 \mathrm{~cm}$. The upper stream side of the channel was filled with particles to a depth of $10 \mathrm{~cm}$. Water was supplied regularly from the upper end and a debris flow was generated. In the stationary state section, measurements were conducted. Material properties were $\tan \phi \mathrm{s}=0.8$ and $\mathrm{d} 50=0.114 \mathrm{~cm}$. In Itoh [2000], a variable slope channel was used. Its length was $12 \mathrm{~m}$ and its width was $10 \mathrm{~cm}$. Water was supplied regularly from the upper end and particles were supplied from a hopper. In the stationary state section, measurements were conducted. Material properties were $\tan \phi \mathrm{s}=0.8$ and $\mathrm{d} 50=0.218$ and $0.305 \mathrm{~cm}$.

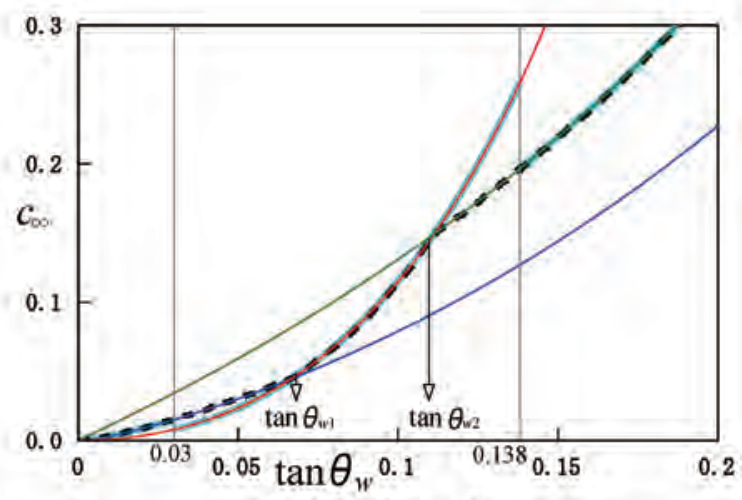

(c) $h / d=30, \varphi_{s}=30$

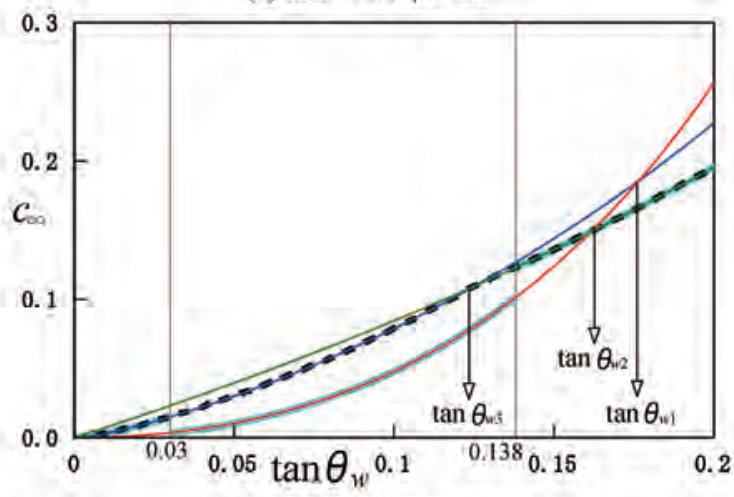

(d) $h / d=30, \varphi_{s}=40$

\subsection{Comparisons}

Figure 4 shows the comparison of the results of the sediment concentration formulae and experimental results of Takahashi [1982]. The results of the sheet flow formula of the Takahashi model and experiments are generally in good agreement. However, the experimental results are slightly larger when $\tan \theta_{w}$ is large. In contrast, the results of the bedload formula agree well with the experimental results. The modified concentration model adopts the bedload formula in this region, so it seems reasonable.

Figure 5 shows the relationship between $h / d$ and $U$ $/ u^{*}$. The experimental results of Takahashi [1982] and Itoh [2000] are divided by gradient. The results of the sheer stress formulae were calculated for each division. Values of $h, n$, and $c_{s}$ are necessary for the calculations. We used $h=0.02$ as the median value of the experimental results $(h=0.01-0.03)$ and $n=0.03$ as a general value. The value of $c_{s}$ was $0.4 c^{*}$ or $0.5 c^{*}$. Calculation results varied with the change in $h$ and $n$; however, the variation was not so large as to affect the following discussion. The experimental results were distributed around the results of the sheet flow formula of Takahashi's model. However, the experimental results became independent of $h / d$ as the incline became lower even though the sheet flow formula of 


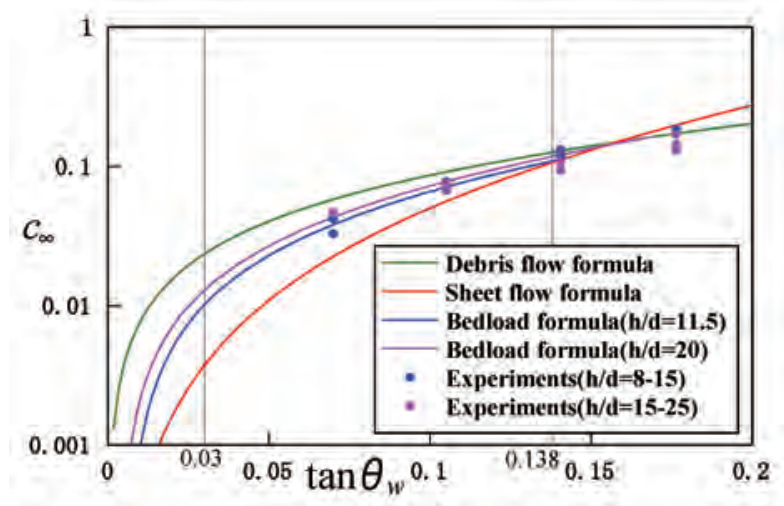

Fig. 4 Comparison of equilibrium sediment concentration

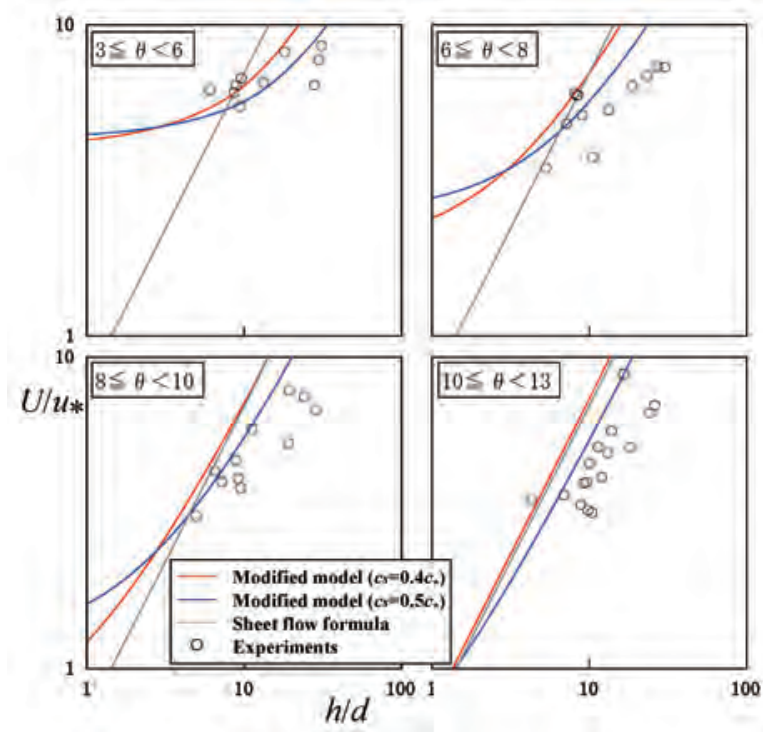

Fig. 5 Comparison of velocity coefficient

Takahashi's model is a function of $h / d$. Results of the modified sheer stress formula agreed well with the experimental results even at low gradients. This was because the modified sheer stress formula was dominated by the water layer at low gradients, which is independent of $h / d$. For the value of $c_{s}, 0.5 c^{*}$ seemed to be better. However, when $h / d$ was large, the calculated results were slightly larger than the experimental results. This is thought to be due to the issue of the applicability of constitutive equations of debris flow, because the constitutive equations of debris flows were derived with an assumption of laminar flow, whereas debris flow may be turbulent when $h / d$ is large. Exploration of this issue will be the subject of future work.

\section{NUMERICAL SIMULATIONS}

\subsection{Methods and Conditions for Numerical Simulations}

To investigate the effectiveness of the modified models, numerical simulations were conducted for deposition processes with changes in riverbed gradient. First, we examined the relationship between the discontinuities and the deposition profiles. Next, we investigated the effects of the discontinuities at the transitional points of flow types on the stability of numerical simulations.

A leap-flog scheme was used in the calculations. Figure 6 shows the initial bed condition and Table 1 lists the parameters used in the simulations. The degree of riverbed gradient decreased gradually from 16.7 at the upper end to 0.38 at the lower end. Spatial resolution, $\Delta \mathrm{X}$, was $5 \mathrm{~nm}$ and the number of calculation points was 300 . The time step of the calculation, $\Delta t$, was $0.1 \mathrm{~s}$. Debris flow at $q=10 \mathrm{~m}^{2} / \mathrm{s}$ was supplied regularly from the upper end. The sediment concentration of the supplied debris flow was equal to equilibrium sediment concentration. In all cases, $d=0.1, \sigma=2.6, \rho=1.0, c^{*}=0.6$, and $n=0.03$ were

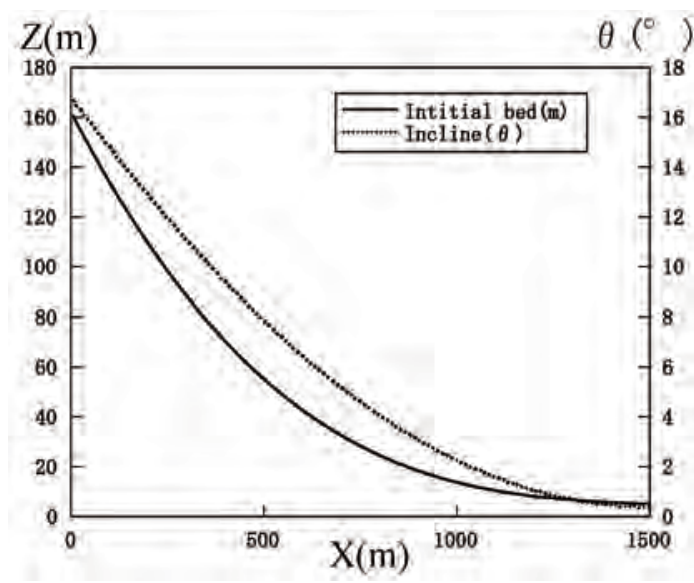

Fig. 6 Initial bed condition

Table 1 Conditions of the simulations

\begin{tabular}{|c|c|c|}
\hline & \\
\hline$\sigma\left(\mathrm{g} / \mathrm{cm}^{3}\right)$ & 2.6 & \\
\hline$\rho\left(\mathrm{g} / \mathrm{cm}^{\prime}\right)$ & 1.0 & \\
\hline$c=$ & 0.6 & \\
\hline$d(\mathrm{~m})$ & 0.1 & \\
\hline$q\left(\mathrm{~m}^{2} / \mathrm{s}\right)$ & 10.0 & \\
\hline$\Delta \mathrm{X}(\mathrm{m})$ & 5.0 & \\
\hline$\Delta \mathrm{t}(\mathrm{s})$ & 0.1 & \\
\hline Case. & Case. 3 & Case. 4 \\
\hline 30 & 35 & 35 \\
\hline 0.54 & 0.46 & 0.46 \\
\hline 0.24 & 0.30 & 0.24 \\
\hline 0.004 & 0.004 & 0.05 \\
\hline
\end{tabular}


fixed conditions. We set four cases with different values of $c_{s}, \phi_{s}, \delta_{e}$, and $\delta_{d}$ to correspond with the discontinuities in Takahashi's model, as indicated in 2.2. Case 1 corresponds to Fig. 2 (a) and 2(b) and Fig. 3 (a) and $\mathbf{3}$ (b). In this case, common parameters were used and only the boundary of bedload and sheet flow was discontinuous in both sheer stress and equilibrium sediment concentration. Case 2 has a different $\phi_{s}$ from Case 1 and corresponds to Fig. 3 (c). In this case, the equilibrium sediment concentration formulae were discontinuous at the boundary of debris flow and sheet flow, as in Case 1. Case 3 has a different cs from Case 1 and corresponds to Fig. 2 (d). In this case, the sheer stress formulae are discontinuous at the boundary of debris flow and sheet flow, as in Case 1. In Cases 1-3, both $\delta_{e}$ and $\delta_{d}$ were 0.004 . The value of $\delta_{d}=0.004$ is less than that of past studies [e.g., Takahashi et al., 1991]. However, we used 0.004 so that some amount of sediment reached the boundary of sheet flow and bedload. Case 4 has larger $\delta_{e}$ and $\delta_{d}$ than Case 1 . We set this to decrease the amount of sediment that reaches the boundary of sheet flow and bedload.

In all cases, numerical simulations were conducted using four methods. The first was the Takahashi model, the second consisted of the modified sheer stress model and the concentration formulae of the Takahashi model, the third consisted of the sheer stress formulae of Takahashi's model and the modified concentration model, and the fourth method consisted of the modified sheer stress and the modified concentration model (the Takahashi, the $\tau_{0} \bmod$, the $c_{\infty} \bmod$, and the $\tau_{0}-c_{\infty} \bmod$ models, respectively).

\subsection{Results and Discussion}

First, we compared the results of deposition profiles. Figures 7 and 8 show the results of deposition height, $\mathrm{dz}$, at 1,000 s for Cases 1 and 2. Figures 7 (a) and 8 (a) are overall views and the results of the Takahashi model and the $\tau_{0}-c_{\infty} \bmod$ model. Figures 7 (b) and 8 (b) are enlarged views of the lower stream $(X=1,000-$ $1,500 \mathrm{~m}$ ) and show the results of all methods on a logarithmic axis. Overall, the results of the four cases are similar; however, there are some differences at specific points due to the discontinuities. In Case $1\left(\phi_{\mathrm{s}}\right.$ $=35$ ), the results of the Takahashi model and the $\tau_{0}$ mod model show sudden changes in deposition height at $\mathrm{X}=1,100 \mathrm{~m}$, because the equilibrium sediment concentration formulae of sheet flow and bedload are discontinuous in Takahashi's model (Fig. 3(a), (b)). The results of the $c_{\infty} \bmod$ model were smooth at $\mathrm{X}=$ $1,100 \mathrm{~m}$ but irregular at $1,350 \mathrm{~m}$ because the sheer stress formulae of Takahashi's model are discontinuous at $c=0.01$. The results of the $\tau_{0}-c_{\infty} \bmod$ model are entirely reasonable. In Case $2\left(\phi_{s}=30\right)$, the results of the Takahashi model and the $\tau_{0}$ mod model show irregular changes in deposition height at $X=500 \mathrm{~m}$ because the equilibrium sediment concentration formulae of debris flow and sheet flow are discontinuous in Takahashi's model, as in Case 1 (Fig. 3 (c)).

Next, we examined the time change in flow height and sediment concentration at the transitional points of the flow types. In the following figures, the results of the four methods are shown. The spots shown in the figures may differ among the methods because they were extracted to correspond to the sediment concentration boundary of debris flow and sheet flow $\left(c=c_{s}\right)$ or sheet flow and bedload $(c=0.01)$. Figures 9 and 10 show the results of Cases 1 and 3. They differ in $c_{s}$. In Case $1\left(c_{s}=0.4 c^{*}\right)$, the results of the flow height in the Takahashi model and the $c_{\infty}$ mod model show slight, irregular variation at the boundary of debris flow and sheet flow because there is a slight discontinuity (Fig. 2 (a), (b)). In Case $3\left(c_{s}=0.5 c^{*}\right)$, the irregularity in the variation in flow height became marked because the discontinuity was larger. In both cases, the $\tau_{0} \bmod$ and $\tau_{0}-c_{\infty} \bmod$ models resolve these problems.

Focusing on the results around the boundary of sheet flow and bedload in Fig. 9 and 10, the flow height variations in Takahashi's model and the $c_{\infty} \bmod$ model show numerical oscillation due to discontinuities in the sheer stress formulae (Fig. 2(a), (b)). Moreover, the numerical oscillation in the Takahashi model is larger than that in the $c_{\infty} \bmod$ model. This is because there is also a discontinuity in the equilibrium sediment concentration formulae in Takahashi's model, which amplifies the oscillation with the discontinuity in the sheer stress formulae. These results for the lower stream are almost in agreement in both cases; that is, the differences in the upper stream do not propagate to the lower stream.

Figure 11 shows the results of Case 4, with larger values of $\delta_{e}$ and $\delta_{d}$. In this case, the sediment concentration keeps the values around equilibrium for long periods of time because the amount of excess sediment reaching this region becomes lower. This means that the duration of the amplification of numerical oscillation becomes longer. The results of Takahashi's model show large oscillations in both flow height and sediment concentration. Numerical oscillations in the $\tau_{0}$ mod model were smaller than those in the Takahashi model because the equilibrium sediment concentration formulae are continuous. The $c_{\infty} \bmod$ and $\tau_{0^{-}} c_{\infty} \bmod$ models resolve these numerical oscillations.

\section{CONCLUSIONS}

In this study, we developed a stable simulation 
International Journal of Erosion Control Engineering Vol. 10, No. 4, 2018

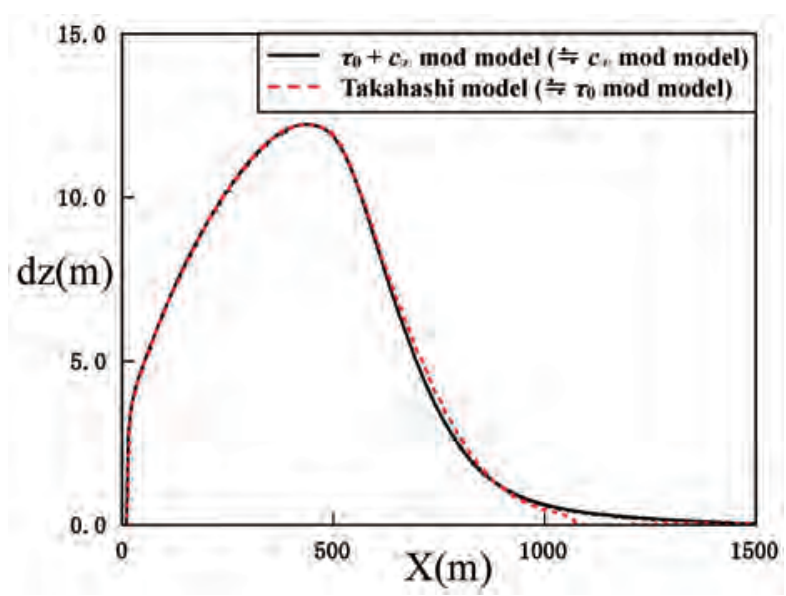

Fig. 7 (a) Deposition depth after 1,000 s (Case 1)

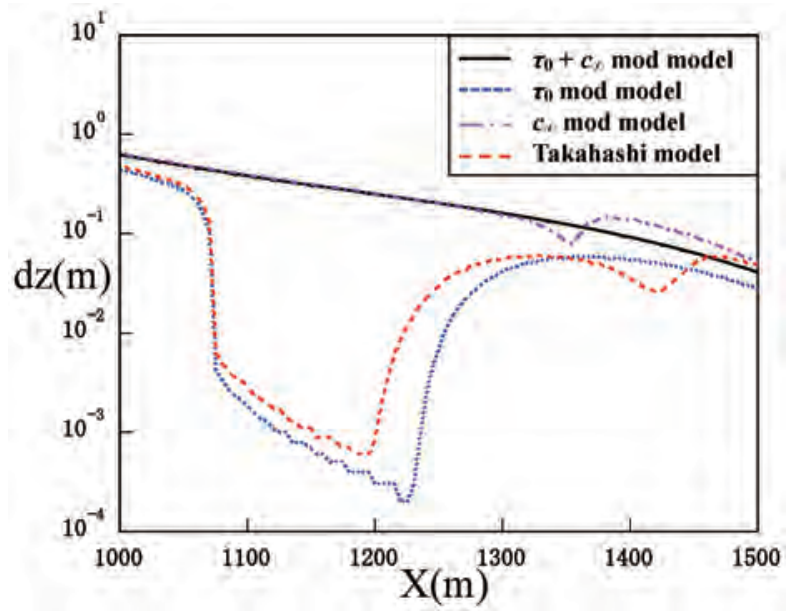

Fig. 7 (b) Deposition depth after 1,000 s (Case 1)

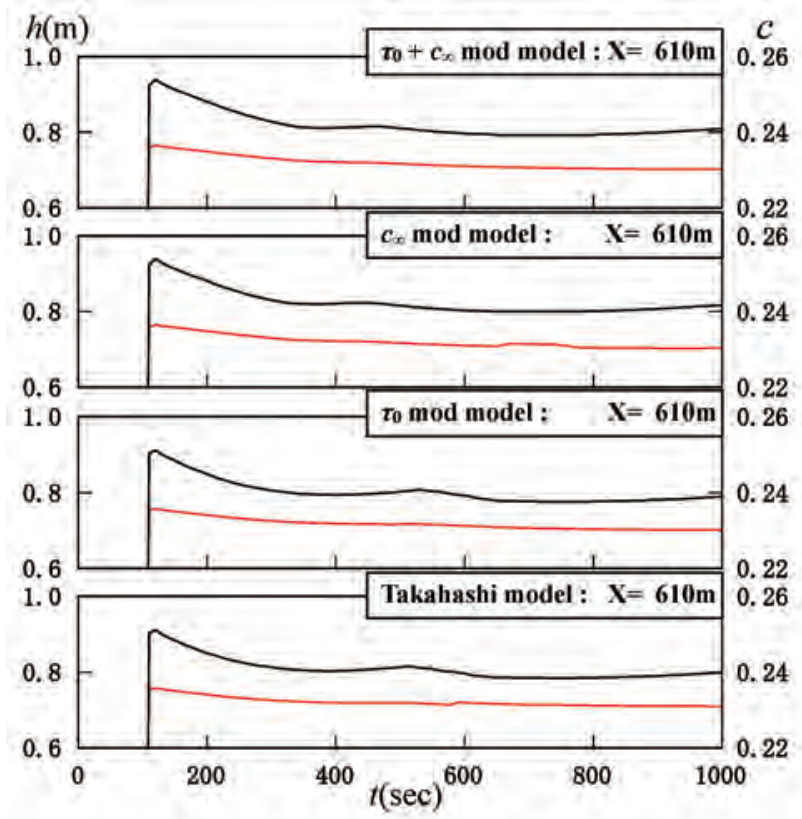

(a)

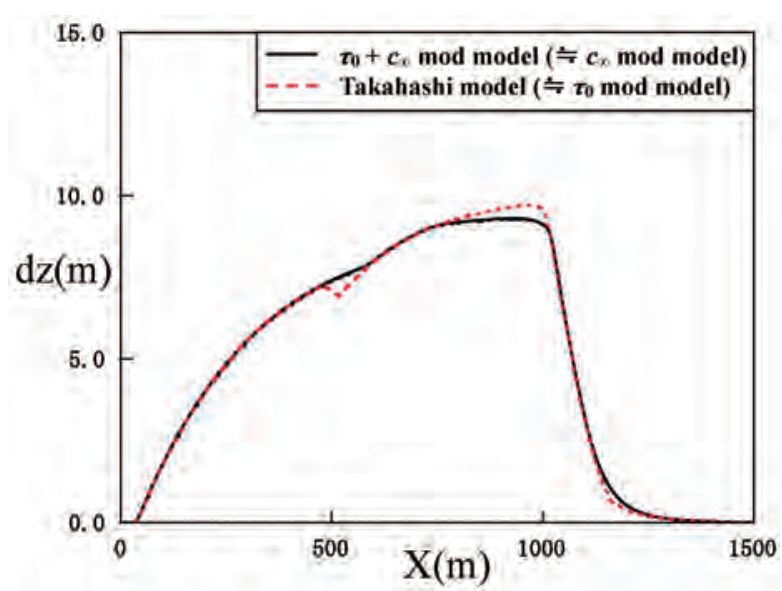

Fig. 8 (a) Deposition depth after 1,000 s (Case 2)

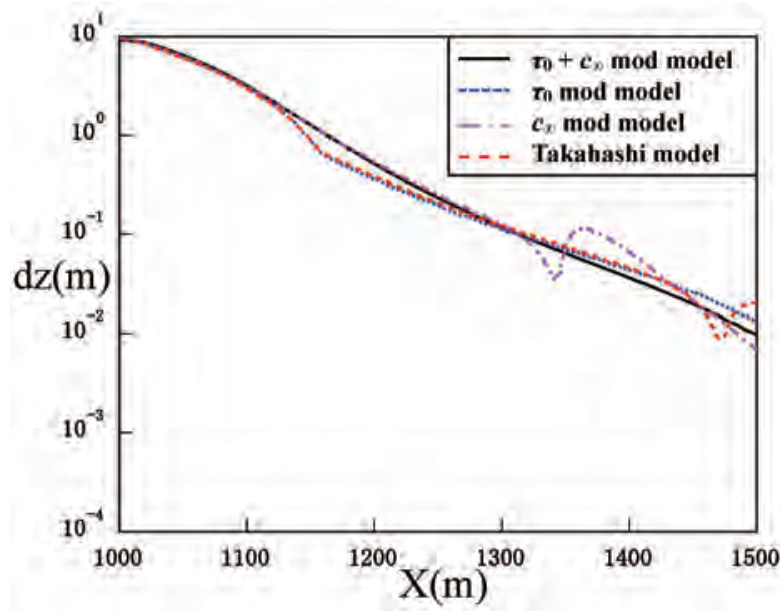

Fig. 8 (b) Deposition depth after 1,000 s (Case 2)

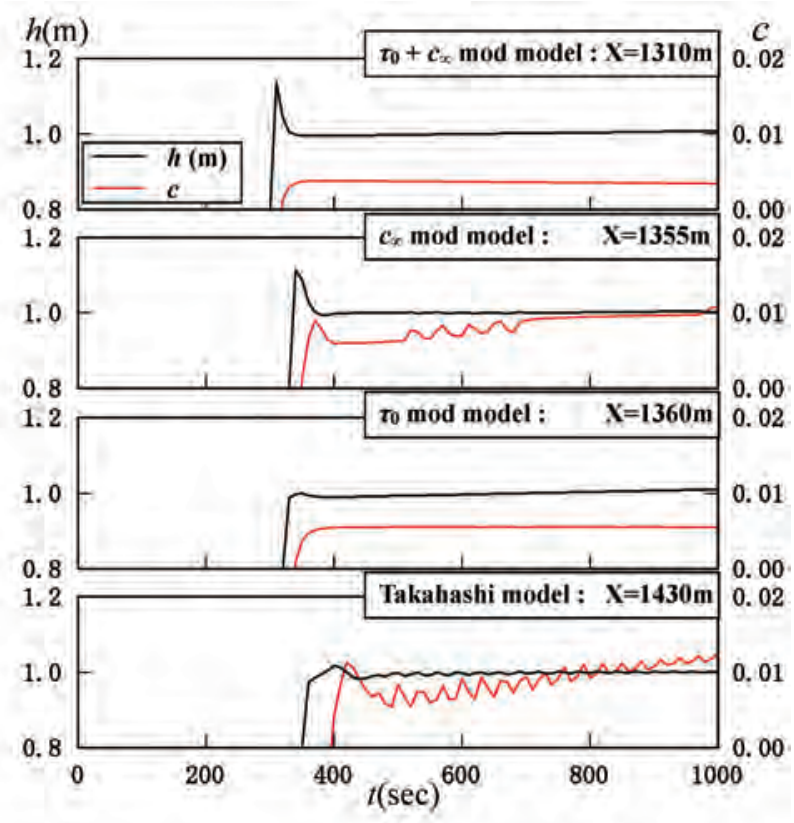

(b)

Fig. 9 Variation in flow depth and sediment concentration with time (Case 1) 


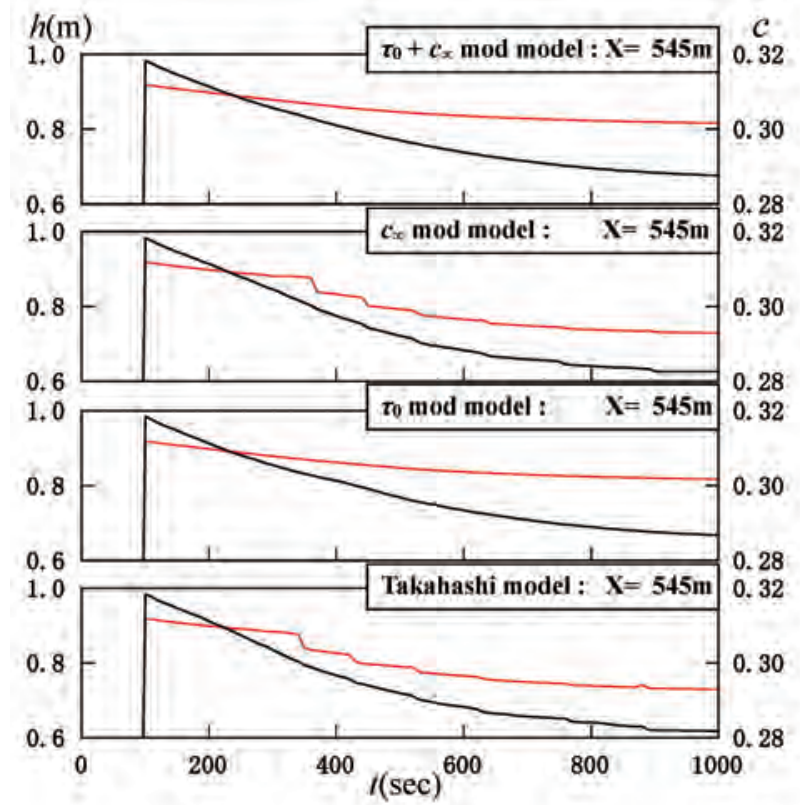

(a)

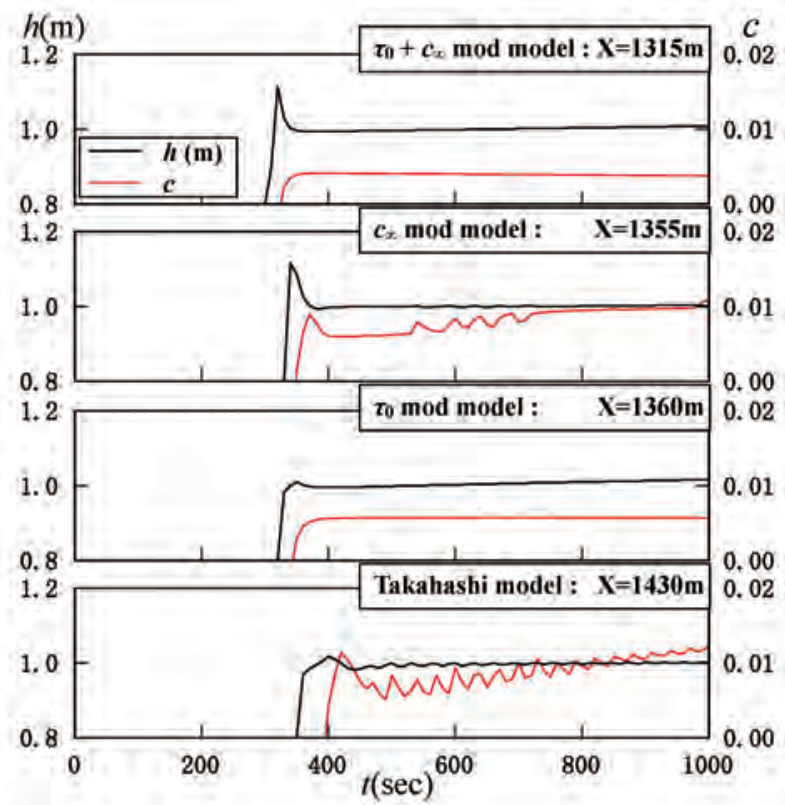

(b)

Fig. 10 Variation in flow depth and sediment concentration with time (Case 3)

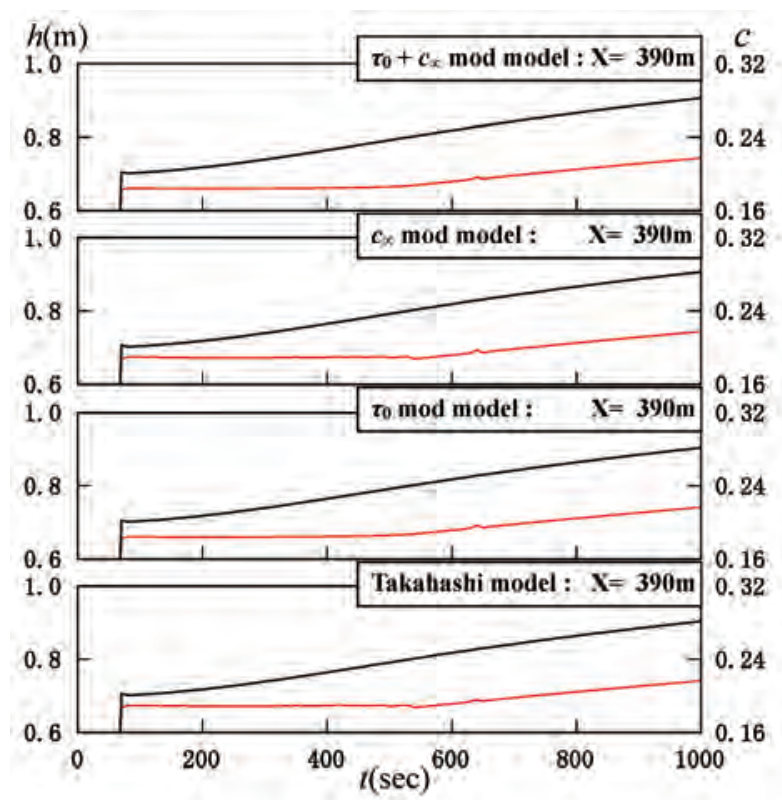

(a)

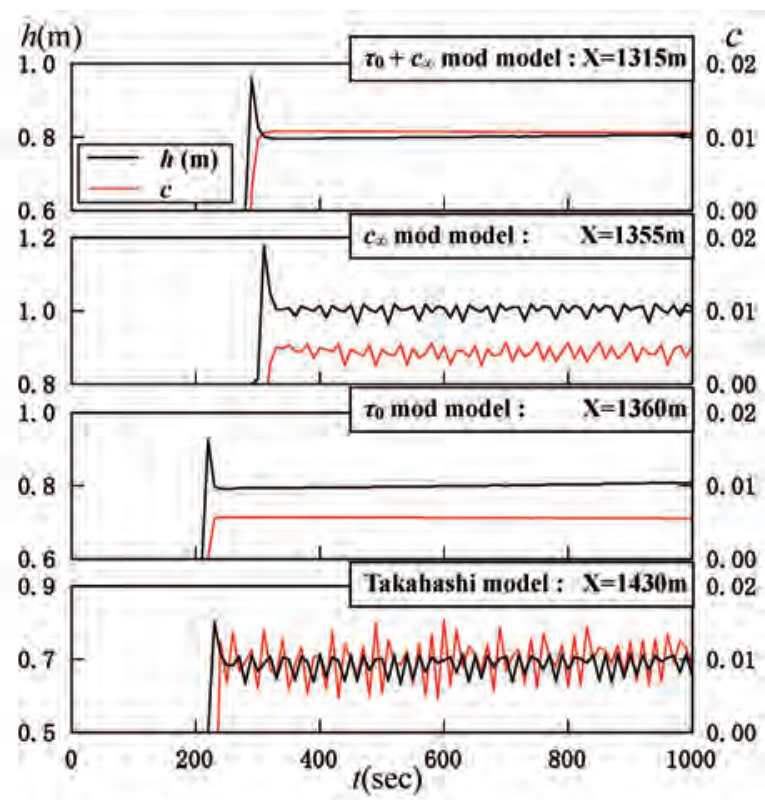

(b)

Fig. 11 Variation in flow depth and sediment concentration with time (Case 4)

method for transitional flow from debris flow through sheet flow to bedload flow by modifying the discontinuities in Takahashi's model. For the sheer stress formula of sheet flow, we adopted the two-layer model for the water layer and the sediment layer, based on past studies. Constitutive equations of debris flow were used for the sediment layer and the Manning equation was applied to the water layer. In the modified concentration model, the boundary conditions were not constant, but varied for continuous transactions.

The modified sheer stress and concentration models were examined by comparison with experimental results from past studies around the region of sheet flow. To investigate the stability of the modified model, numerical simulations were conducted for deposition processes with changes in riverbed gradient. 
The conclusions from this study can be summarized as follows.

Comparison with experimental results

(1) Calculated results for the equilibrium sediment concentration with Takahashi's model were slightly lower than the experimental results when the riverbed gradient was low. In contrast, the results of the modified concentration model agreed well with the experimental results.

(2) The velocity coefficient calculated using the sheet flow formula of Takahashi's model disagreed with experimental results when the riverbed gradient was low. The results of the modified sheer stress formula agreed well with experimental results.

Results of numerical simulations

(3) Overall, the results of Takahashi's model and the modified model were similar in deposition area.

(4) The profiles of deposition depth showed irregular forms when the equilibrium sediment concentrations were discontinuous.

(5) The profiles of deposition depth showed irregular forms when the riverbed sheer stress formulae were discontinuous.

(6) Numerical oscillations in time change of flow depth occurred when the riverbed sheer stress formulae were discontinuous.

(7) In addition to (6), when the equilibrium sediment concentration formulae were also discontinuous, numerical oscillations were amplified.

(8) The modified model could yield smooth and stable simulation results without these problems.

We could confirm high agreement with the experimental results and numerical stability of the modified model, as above. The Takahashi model requires a low time step for calculations because numerical oscillations may cause numerical divergence. High stability of the modified model enabled shorttime calculations with a large time step.

The modified method in this study is an improved method for widely used bed deformation calculations. We cannot use the model for exact simulations, like that of the vertical distribution of sediment concentration, because the Manning equation is a formula of average velocity. Our model is limited for depth-integrated simulations. Moreover, it may be unreasonable that the Manning coefficient affects the water layer even when the depth of sediment layer is large. This issue should be investigated further, but is not serious because the velocity coefficients of sheet flow are certainly intermediate between debris flow and bedload.

\section{REFERENCES}

Egashira S., Ashida K., Takahama J. and Tanonaka S. (1990) : Sediment Transport Formula Derived From An Energy Dissipation Model, Annuals, Disas. Prev. Res. Inst., Kyoto Univ., No. 33 B-2, pp. 293-306 (in Japanese)

Egashira S., Miyamoto K., and Itoh T. (1997) : Bed-load Rate In View Of Two Phase Flow Dynamics, Annual Journal of Hydraulic Engineering, JSCE, Vol. 41, pp.789-794(in Japanese with English abstract)

Itoh T. (2000) : Study on Constitutive Equations of Debris Flow and Their Applicability, Ph.D. thesis, Ritsumeikan Univ.

Japan Society of Erosion Control Engineering (2000) : Simulation Method for bed deformations in mountain rivers, Sankaido, $143 \mathrm{p}$

Miyamoto K. and Ito T. (2002) : Numerical simulation method of debris flow introducing the erosion rate equation, Journal of the Japan Society of Erosion Control Engineering, Vol. 55, No. 2, pp. 24-35 (in Japanese with English abstract)

Nakagawa H., Takahashi T., Sawada T. and Satofuka Y. (1990) : Design Hydrograph and Evacuation Planning for Debris Flow, Annuals, Disas. Prev. Res. Inst., Kyoto Univ., No. 39 B-2, pp. 347-371 (in Japanese)

Nakagawa H., Takahashi T., Satofuka Y. and Kawaike K. (2001) : Sediment Disasters Caused by the Heavy Rainfall in the Camuri Grande River Basin, Venezuela, 1999. Reproduction of Sediment Runoff, Flooding, and Deposition and Evaluation of Effectiveness of the Sabo Works by Means of Numerical Simulation-, Annuals, Disas. Prev. Res. Inst., Kyoto Univ., No. 39 B-2, pp. 347-371 (in Japanese)

Nakatani K. (2010) : Development and Application of GUI Equipped Universal Simulation System of Debris Flow, $\mathrm{Ph}$. D. thesis, Kyoto Univ. (in Japanese)

Takahama J., Fujita Y., and Kondo Y. (2000) : Analysis Method Of Transitional Flow From Debris Flow To Sediment Flow, Annual Journal of Hydraulic Engineering, JSCE, Vol. 44, pp. 683-686 (in Japanese with English abstract)

Takahashi T. (1982) : Study on the Deposition of Debris Flows (3) - Erosion of Debris Fan-, Annuals, Disas. Prev. Res. Inst., Kyoto Univ., No. 25 B-2, pp. 327-348 (in Japanese with English abstract)

Takahashi T. (2004) : Debris Flow: Mechanics, Prediction and Countermeasures, Kinmiraisha, pp. 88-89

Takahashi T. and Nakagawa H. (1991): Prediction of Stony Debris Flow Induced by Severe Rainfall, Journal of the Japan Society of Erosion Control Engineering, Vol. 44, No. 3, pp. 47-52 (in Japanese)

Takahashi T., Nakagawa H., and Sato H. (1988) : Fan Foundation Due to the Runoff of the Debris and Immature Debris Flows, JSCE, Vol. 32, pp. 497-502 (in Japanese)

Received : 29 June, 2016

Accepted : 20 July, 2016

This article was translated from an original article published in the Journal of the Japan Society of Erosion Control Engineering. 